\title{
Intervención psicoterapéutica integrativa en un caso de celotipia
}

\section{Integrative psychotherapeutic intervention in a case of cellotipia}

Aarón Israel Pacheco-Miranda

Manuel Sosa-Correa

Elia María Escoffié-Aguilar

Universidad Autónoma de Yucatán

\section{Resumen}

El presente estudio tiene como objetivo mostrar la intervención en un caso de celotipia desde un enfoque integrativo de técnica de orientación y teórica amplia, donde se conjuntan los enfoques cognitivoconductual y psicodinámico. El diagnóstico se efectuó utilizando técnicas de ambos enfoques; se incluyeron las pruebas proyectivas Machover y Test de la figura bajo la lluvia y el cuestionario de esquemas mal adaptativos, así como el Manual Diagnóstico y Estadístico de los Trastornos Mentales DSM-V (APA, 2013). Se efectuaron 30 sesiones terapéuticas, y se encontraron factores psicógenos que explican la aparición y mantenimiento de síntomas celotípicos. Se realizó la intervención, integrando ambas corrientes al servicio del paciente con resultados positivos, y se consiguió la remisión de la mayoría de los síntomas reportados e inferidos y la toma de conciencia de las causas subyacentes de su celotipia, así como la detección de las cogniciones que la mantenían.

Palabras clave: celotipia, psicoterapia cognitiva, psicoterapia psicoanalítica, enfoque integrativo, estudio de caso.

Nota del autor

Aarón Israel Pacheco-Miranda, Facultad de Psicología, Universidad Autónoma de Yucatán (UADY). La correspondencia en relación con este artículo debe dirigirse a Aarón Israel Pacheco-Miranda, Facultad de Psicología, UADY. Carretera Mérida Tizimin S/N, Cholul C.P. 97305, Mérida, Yucatán, México.

Dirección electrónica: psicaron@gmail.com 


\begin{abstract}
This case study aims to intervene in a case of cellotipia from a "integrative approach of technical integration of orientation and theoretical integration broad", combining the cognitive-behavioral and psychodynamic approaches with the clinical case study modality. The diagnosis was made using techniques from both approaches, including the Machover projective tests and the Test of the figure in to the rain and the questionnaire of maladaptive schemes, as well as the Diagnostic and Statistical Manual of Mental Disorders DSM-V (APA, 2013). Thirty therapeutic sessions were performed; psychogenic factors were found that explained the appearance and maintenance of cellopathy symptoms. The intervention was performed integrating both streams to the service of the patient with positive results, the remission of the majority of the reported and inferred symptoms was achieved and awareness of the underlying causes of its cellotipia, as well as stop the congnitions that kept it.
\end{abstract}

Keywords: cellotipia, cognitive psychotherapy, psychoanalytic psychotherapy, integrative approach, case studies.

Los celos son una emoción que tiene su origen en un deseo desmedido por poseer algo de forma exclusiva, subyace a la idea de la infidelidad (real o imaginaria) de la persona que se ama. Los celos se encuentran condicionados por un sentido desmesurado de propiedad y de exclusividad, más allá del exclusivo deseo sexual (Caudillo \& Cerna, 2007).

En la personalidad celosa es frecuente encontrar vulnerabilidad psicológica. Así, por ejemplo, en una relación de pareja hay que aceptar cierto grado de incertidumbre en relación a la posible pérdida o abandono del otro. Por lo tanto, un factor de vulnerabilidad sería una necesidad extrema de certeza unida a una necesidad de control de la persona amada. Estos dos factores nucleares, en el inicio y mantenimiento del problema, entroncarían directamente con la historia de aprendizaje de la persona. (Cuesta-Bayón, 2006).
Existen celos considerados normales o adaptativos, frecuentes entre la población, que no constituyen un trastorno psicopatológico. Hasta cierto punto, pueden reflejar interés y el amor que la persona siente hacia su pareja; tienen un valor de adaptación evolutiva, puesto que aseguran la estabilidad de la pareja, del hogar, y crean un ambiente apropiado para la crianza de los hijos, además, sirven como antídoto para la promiscuidad que, a la larga, pudiera generar inestabilidad familiar (González \& Herrera, 2002). Buss y Schmittmith (1993) entienden los celos en términos de estrategias de afrontamiento que permiten mantener $y$ consolidar las parejas. Por ende, los celos sanos consisten en una preocupación por la posible pérdida de una persona amada o malestar por la relación real o imaginada que esa persona tiene con alguien más. 
Con respecto al tema central de esta investigación, López (2002), Martínez-León y colaboradores (2013) afirman que los celos patológicos son un trastorno, producto de una alteración afectiva-emocional anormal, que activa conductas moldeadas por estados afectivos, donde varias funciones psicológicas se alteran profundamente. De ahí que la celotipia se considere la manifestación de celos patológicos y compulsivos. La persona que la padece manifiesta serias alteraciones en ámbitos de pareja familia/o laborales. La persona con celotipia busca confirmar la creencia de que su pareja le es infiel con base en conclusiones erróneas, apoyadas por pequeñas pruebas, por ejemplo, manchas en las sábanas, olores, llamadas telefónicas, etc. Después, el sujeto con celotipia intenta intervenir en la infidelidad imaginada, ya sea investigando al supuesto amante o agrediendo a la pareja.

Las personas con celotipia pueden desarrollar un estado de ánimo irritable, les enoja todo o se comportan de manera agresiva con su pareja o cónyuge, también pueden tener accesos de ira o comportamiento violento. La celotipia es definida como un subtipo de trastorno delirante, presente cuando "el tema central del delirio del individuo es que su cónyuge o amante le es infiel" (APA, 2013, p. 50).

A continuación, se establecerán las consideraciones principales que al respecto tienen los enfoques psicológicos utilizados en el presente estudio, iniciando con el psicoanálisis, cuya teoría da una explicación muy completa de la celotipia.

Bowlby (1982, citado en Caudillo \& Cerna, 2007) explica que los celos se dan cuando la persona siente amenazada su seguridad, la cual es requerida para un adecuado desarrollo en la primera infancia y llega a ser tan importante como el alimento mismo. Por lo tanto, las personas conservan la necesidad de apegarse a una figura que les brinde seguridad toda la vida. Así, la madre, figura del apego inicial, puede verse sustituida por otra persona, por ejemplo, la pareja en la vida adulta. Con respecto a este tipo de pacientes, Kernberg (1995) establece que son incapaces de integrar la agresión con el amor, es decir, de poner los componentes agresivos y sadomasoquistas de la sexualidad infantil al servicio de la gratificación erótica libidinal. Puesto que esta integración de la excitación sexual y el deseo erótico se produce antes de que el sujeto tenga capacidad para integrar las relaciones objetales internalizadas e investidas de manera libidinal.

Para Freud (1955) tanto los celos como la tristeza son estados afectivos que pueden considerarse normales. De este modo, cuando parecen afectar el carácter y la conducta de un individuo, se deduce con justificación que han sucumbido a una enérgica represión y desempeñan, por consecuencia, en su vida anímica inconsciente, un papel más importante. Los casos de celos anormalmente intensos, observados en el análisis, muestran tres distintos 
estratos o grados que se pueden clasificar en la siguiente forma: celos concurrentes o normales; celos proyectados; y celos delirantes.

Los celos concurrentes o normales se componen, en esencia, de la tristeza y el dolor por el objeto erótico que se cree perdido, de la ofensa narcisista en cuanto nos es posible diferenciarla de los elementos restantes y, por último, de sentimientos hostiles contra el rival preferido, además de una aportación más o menos grande de autocrítica que quiere hacer responsable al propio yo de la pérdida amorosa.

Los celos del segundo grado, o celos proyectados, nacen de las propias infidelidades del sujeto o del impulso por cometerlas, relegado éste a lo inconsciente por la represión. Precisamente, aquellos que niegan experimentar tales impulsos sienten su presión de manera enérgica que suelen acudir a la proyección como mecanismo inconsciente para aliviarla, y alcanzan tal alivio e incluso una absolución completa por parte de su conciencia moral, al proyectar sus propios impulsos de infidelidad sobre la persona a quien deben de proteger.

Por su parte, los celos del tercer grado o delirantes, tienen origen en tendencias infieles reprimidas, pero los objetos de las fantasías son de carácter homosexual, ya que corresponden a una homosexualidad inconsciente y proyectada en la pareja y ocupan con pleno derecho un lugar entre las formas clásicas de la paranoia. Como tentativa de defensa contra un poderoso impulso homosexual masculino, podrían ser descritos por medio de la siguiente fórmula: "No soy yo quien le ama, es ella". Es un caso de celos delirantes pueden encontrarse celos de los tres grados y no sólo del tercero.

Durante un tratamiento psicoanalítico se presentan muchas variables en consideración. A continuación, se mencionarán varias que atañen directamente al caso analizado en el presente artículo. Para el psicoanálisis, el término "relaciones de objeto" se refiere a una estructura intrapsíquica y no a relaciones externas interpersonales, lo cual no impide que dichas estructuras se exterioricen en relaciones interpersonales. Al invertir la frase, se puede decir que en toda relación interpersonal no puede manifestarse otra cosa que esta estructura intrapsíquica. Es decir, el mundo interno de las relaciones objetales, modula el trato del sujeto con los otros en el mundo externo (EsquivelSosa \& Gómez-Castillo, 2011).

Los elementos propios de este "mundo interno" se establecen por medio de la internalización, la cual puede efectuarse promovida por el amor y el deseo de guardar algo bueno dentro de sí, o bien, bajo la influencia de devorar de manera agresiva, comer y morder destruyendo. En el primer caso, el objeto internalizado es una fuente de protección y ayuda, también puede entrar a formar parte del yo y contribuir a su desarrollo y crecimiento. Cuando, por el contrario, el objeto ha sido internalizado de forma devoradora, se convierte en hostil y agresivo, por consiguiente, 
en la fantasía el sujeto es vivenciado como un enemigo que perturba el funcionamiento psíquico y corporal (Coderch, 1990).

Entre los objetivos primordiales de la psicoterapia psicoanalítica se encuentra que el paciente logre sublimar -este concepto fundamental lo aborda Nasio (1996) -, como un medio de transformar y de elevar la energía de las fuerzas sexuales, convirtiéndolas en una fuerza positiva y creadora. Para este autor, Freud, desde los inicios de su obra, consideró la sublimación como una de las defensas del yo contra la irrupción violenta de lo sexual o como uno de los modos de defensa que se oponen a la descarga directa y total de la pulsión.

Greenson (2004) establece que un análisis psicoanalítico busca la traslaboración como una manera de conseguir cambios significativos en la vida del paciente. La traslaboración es una compleja serie de procedimientos y procesos que se produce después de presentarse un insight (fenómeno cognitivo en el cual se llega a la solución o comprensión de un conflicto en asociación con una vivencia afectiva de sorpresa y certeza ante el descubrimiento [Palma \& Cosmelli, 2008]), y consiste en exploraciones repetitivas, progresivas y elaboradas de las resistencias que dificultan los cambios antes mencionados.

Desde la perspectiva cognitiva-conductual, al igual que se mencionó con Freud (1955), los celos como cualquier emoción puede tener una función adaptativa, siendo que su uso desadaptativo se da cuando esta emoción, dificulta o impide a la persona el logro de sus objetivos (Sosa-Correa, Navarrete-Centeno, \& Escoffié-Aguilar, 2014).

Quienes sienten celos de manera adaptativa pudieran preferir que sus parejas permanezcan con ellos y no desear que tengan una relación demasiado íntima con nadie más. Esto a veces causa algunos problemas en la pareja, pero no son demasiado serios ni producen un malestar intenso en ninguno de los miembros. Por el contrario, los celos patológicos o disfuncionales vienen de creencias irracionales, demandantes y rígidas (Ellis \& Blau, 2000), y están acompañados de intensos sentimientos de inseguridad, auto-compasión, hostilidad y depresión; asimismo, presentan manifestaciones clínicas en varios niveles: en el plano emocional es frecuente la ansiedad y/o agresividad; en el nivel cognitivo, los pensamientos intrusivos relacionados con la infidelidad; y en el nivel conductual, las demandas de seguridad o rituales compulsivos (i.e., motores y verbales) con el objeto de controlar al cónyuge (Carlen, Kasanzew, \& López, 2009).

Las personas con celos patológicos exigen o demandan que su pareja bajo ningún concepto se implique emocional o sexualmente con otras personas. Al estar utilizando un pensamiento rígido, basado en exigencias absolutistas -no admiten más posibilidad que el cumplimiento de sus deseos-, perciben la posibilidad de una infidelidad como algo terrible (Ellis \& Blau, 2000). En cambio, cuando una persona utiliza un pensamiento flexible, se dice a sí misma 
cosas como: "Deseo mucho que mi pareja esté sólo conmigo, pero es libre de elegir lo que quiere, y si me deja será doloroso y frustrante, pero no será algo terrible, ni insoportable, ni me matará".

La persona que piensa de este modo puede estar preocupada, pero no aterrorizada ante la posibilidad del abandono, ni necesitará estar constantemente en guardia por si sucede eso tan temido. En cambio, si está utilizando una creencia irracional o pensamiento demandante, rígido y dogmático, creerá cosas como: "Mi pareja no puede ni debe dejarme nunca bajo ninguna circunstancia ni tiene derecho a hacerlo porque si lo hace me sentiré fatal y será terrible e insoportable". La persona que piensa de este modo se sentirá muy ansiosa, insegura, deprimida, agresiva y dependiente (HuertaHernández \& Alcázar-Olán, 2014).

\section{Método}

Se eligió el estudio de caso como método para estudiar a profundidad este fenómeno. Se abordó desde una postura integrativa de técnica de orientación y teórica amplia, que consiste en crear un marco teórico específico para, posteriormente, establecer una metodología conformada por el planteamiento del problema y elaboración de los objetivos planteados. Tal proceder permite utilizar instrumentos y técnicas psicoterapéuticas de cualquier tipo, en este caso, los enfoques psicoanalítico y cognitivoconductual (Rosado-Rosado, Vázquez-Vargas,
\& Cetina-Sosa, 2016). Por su parte, el objetivo de este estudio fue mostrar la eficiencia de una intervención en un caso de celotipia desde un enfoque integrativo de técnica de orientación y teórica amplia, conjuntando los enfoques cognitivo-conductual y psicodinámico.

\section{Participantes}

Un adulto joven de 24 años, que vive con sus padres y hermanos, a quien se le llamará Diego (se cambia su nombre y el de su pareja para garantizar el anonimato del participante), pasante de Ingeniería Química. En la actualidad, mantiene una relación de pareja, desde hace seis años, con Elena, quien es de su edad y trabaja como secretaria de una dependencia de gobierno. Desde el inicio de su relación, la pareja ha mantenido una dinámica de dependencia y disfuncionalidad. De la entrevista se obtuvo que, desde el punto de vista de Diego, Elena usa las mentiras y engaños para atacarlo o, simplemente, para salirse del control tan férreo al que él la somete, por ejemplo, al revisar sus correos electrónicos, mensajes y registros de llamadas, administra su sueldo, la vigila con frecuencia $\mathrm{y}$, en general, no le permite tener espacio personal. Elena mantiene una actitud desafiante y ambivalente ante Diego, acepta su control, pero miente para salirse de él. Ella ha admitido un par de infidelidades, de modo que al confrontarse con Diego, ambos han llegado a recurrir a la agresión física de manera constante.

\section{Instrumentos}

Cuestionario de las Trampas Vitales de Young y Klosko: escala tipo Likert con seis opciones de 
respuesta. Sirve para determinar los esquemas mal adaptativos que el sujeto posee (Young \& Klosko, 2003), los cuales son constructos no probados empíricamente, fenómenos de un nivel profundo en demasía, que pueden estar inactivos durante mucho tiempo y luego ser activados (energizados) con premura, como resultado de cambios en el tipo de estímulos que llegan del ambiente y que afectan significativamente la vida de la persona (Young, 1999).

Test de la figura humana Machover: prueba proyectiva que consiste en dibujar a una persona en una hoja en blanco. Este test es útil porque permite expresar pensamientos, sentimientos, aptitudes, así como una imagen total del sí mismo, incluyendo rasgos permanentes de la personalidad, dimensiones ignoradas, mecanismos de defensa, identidad sexual, entre otras (Xandró \& Belda, 2007).

Test de la persona bajo la lluvia de Querol y Chaves: prueba proyectiva que consiste en que el sujeto dibuje en una hoja en blanco, bajo la siguiente consigna: "dibuje a una persona bajo la lluvia". Esta prueba arroja información importante acerca de la imagen corporal del individuo bajo condiciones ambientales desagradables, tensas, donde la lluvia representa un elemento perturbador (Querol \& Chaves, 2009).

Historia clínica: documento escrito cuya información ayuda a identificar al paciente, sirve para justificar el diagnóstico y el tratamiento, así como para documentar correctamente los resultados obtenidos en anteriores diagnósticos.
Guía de consulta de los criterios diagnósticos del DSM 5: incluye la clasificación diagnóstica completa revisada, además de todos los criterios diagnósticos en un formato fácil de utilizar. Proporciona un acceso rápido a la información esencial para emitir un diagnóstico.

\section{Procedimiento}

El proceso terapéutico se llevó a cabo en un consultorio particular, propio del terapeuta. Se trabajó de manera integrativa durante 30 sesiones de aproximadamente 45 minutos cada una, en la ciudad de Mérida, Yucatán. En la tabla 1 se muestra el diagnóstico, intervención y mantenimiento, sin embargo, se considera importante ahondar en la etapa de diagnóstico, por lo cual a continuación se presentan la integración de pruebas y la conceptualización del caso que se consideran etapas de la misma.

\section{Integración de pruebas.}

\section{Test de la figura humana.}

El paciente presenta una identificación de género masculino, aunque la imagen que presenta sea distorsionada. De acuerdo con la interpretación del test, el tipo de cuello que dibuja es indicativo de tener problemas para controlar los impulsos instintivos; teme que su "yo" se vea desbordado. La omisión de las manos representa una marcado conflicto con afectos relacionados a contacto y manipulación, esto sugiere dificultad para interactuar de manera profunda con otras personas, conflictos con la productividad, sentimientos de culpa por conductas de robo o masturbación. 
La manera en la que los brazos nacen del cuerpo, denota una falta de fortaleza emocional y conflictos con la propia virilidad; el cabello, la forma de la nariz y el remarcado en el área genital reflejan conflictos de índole sexual, ya sea en su identificación, como en la percepción de esta esfera en su vida. La ausencia de pupilas en los ojos puede interpretarse como culpa vouyerista o presencia de rasgos paranoides.

En el segundo dibujo, que fue una mujer, se observa que el sujeto tiene una visión un tanto infantil de las mujeres, a quienes puede considerar pedantes o más inteligentes que los hombres. Las relaciona con un mayor control de impulsos instintivos. Los senos incipientes se relacionan con una marcada dependencia, infantilidad y con un ajuste imperfecto. Las considera poco apegadas a la realidad, evasivas y con reducido contacto social y/o emocional.

\section{Test de la persona bajo la lluvia.}

El análisis de esta prueba mostró, en general, características similares a las del Test de dibujo de la figura humana, reportado anteriormente, a dicha interpretación habrá que agregarle algunos elementos nuevos, como la forma de los zapatos puede indicar un intento por mantenerse en la realidad, de pensar de manera concreta y realista; la gorra puede ser un intento de ocultarse, o bien, en el contexto de estar bajo la lluvia, puede ser un mecanismo infantil de defensa, el paraguas pequeño indica labilidad emocional al enfrentarse a conflictos, dificultades en las relaciones interpersonales y /o con las figuras de autoridad; el mango del paraguas, sombreado, es un indicador de tener la necesidad imperiosa de aferrarse a algo a manera de defensa, aun cuando no esté seguro de que le servirá como tal; los relámpagos indican que los conflictos de su medio lo impactan de manera importante, lo cual se relaciona con la forma en la que dibujó las gotas de lluvia, las cuales son una representación de angustia.

\section{Cuestionario de trampas vitales.}

Este cuestionario se aplicó con el fin de establecer los principales esquemas tempranos y determinar cuáles serían mal adaptativos. Los resultados indicaron que entre los esquemas mal adaptativos encontrados está el esquema de abandono, el de privación emocional y el de subyugación, aunque es necesario ampliar estas hipótesis con la historia clínica.

\section{Historia clínica.}

En la exploración de la historia clínica se pudieron evidenciar tanto los factores condicionantes como los detonantes y reforzadores de los síntomas celopáticos, tanto a nivel familiar como sexual, siendo los primeros los más relevantes; en este caso, la dinámica familiar, condicionada por la infidelidad materna, incidió de manera significativa en la formación tanto de ideas irracionales y rígidas, como de una introyección tóxica de ambos objetos parentales, particularmente de la madre.

\section{DSM V.}

Diego, al momento de iniciar el análisis, presentaba los cinco criterios diagnósticos para los trastornos de tipo delirantes (APA, 2013): 
A. Presencia de uno (o más) delirios de un mes o más de duración.

B. Nunca se ha cumplido el criterio A de esquizofrenia.

C. Aparte del impacto del delirio (s) o sus ramificaciones, el funcionamiento no está muy alterado y el comportamiento no es manifiestamente extravagante o extraño.

D. Si se han producido episodios maníacos o depresivos mayores, han sido breves en comparación con la duración de los períodos delirantes.

E. El trastorno no se puede atribuir a los efectos fisiológicos de una sustancia o a otra afección médica y no se explica mejor por otro trastorno mental, como el trastorno dismórfico corporal o el trastorno obsesivo-compulsivo.

\section{Conceptualización del caso}

El sujeto (Diego) es una persona con rasgos controladores y celopáticos, con así duidad espía, vigila y hace lo posible por coartar la privacidad de su pareja, con quien mantiene una relación de noviazgo desde aproximadamente 6 años. A menudo interpreta cualquier situación ambigua como una prueba fehaciente de la infidelidad de su novia, lo cual le genera episodios de angustia $\mathrm{y}$ violencia psicológica. Esto ha llevado su relación a un estado de desgaste tal, que las agresiones físicas de ella hacia él son comunes $\mathrm{y}$, en cada ocasión, más fuertes, lo cual genera sospechas cada vez mayores. No presenta disfuncionalidad en ninguna otra área, ni ideas delirantes, aparte de las concernientes a la pareja; estas sospechas no se deben a consumo de medicamentos ni alguna otra sustancia que altere su percepción de la realidad.

Tabla 1

Esquematización de las sesiones

Diagnóstico 1 a 5 sesiones

Reestructuración cognitiva

Psicoanálisis

Establecimiento de rapport, inicio de alianza terapéutica, aplicación, calificación e interpretación de las pruebas según la orientación metateórica, explicado en el apartado de integración de pruebas que da lugar a la conceptualización del caso

Establecimiento de motivo manifiesto y latente, análisis inicial de la transferencia y contratransferencia

Establecimiento de rapport. Aclaración del motivo de consulta, objetivos de terapia, inicio de alianza terapéutica
Motivo manifiesto. Diego acude a terapia con la consigna de encontrar la manera de poder "corregir" a su novia, pues considera que ella es muy injusta con él, lo engaña, le miente y, en términos generales, no se comporta como Diego desea que lo haga

Motivo latente. Reparar el trauma vivido en su adolescencia, cuando su madre le fue infiel a su padre con un vecino. Diego fue testigo y en ocasiones encubrió a su madre para evitar un conflicto mayor; desde el inicio de su relación, hace seis años, tiene conductas y pensamientos celotípicos. La celotipia está asociada a impulsos homosexuales reprimidos, idealmente, Diego asumiría la conciencia de estos impulsos 


\section{Tabla 1}

\section{Continuación}

Reestructuración cognitiva

Esta fase fue extensa, pues las ideas irracionales y distorsiones cognitivas que perpetuaban la celotipia se encontraban fuertemente enraizadas; en su adolescencia, Diego presenció varios episodios de infidelidad materna, hecho que le generó un resentimiento muy marcado hacia su madre, que generalizó hacia las mujeres, sin embargo, este resentimiento se manifestaba en forma de desconfianza extrema

La parte fundamental de esta fase fue discutir racionalmente sus creencias irracionales acerca de la situación antes mencionada, así como el actuar de su padre, a quien en secreto, Diego recrimina por su pasividad

Las estrategias terapéuticas se centraron en fortalecer su sentido de la lógica. A menudo, Diego infería de manera arbitraria conclusiones irreales de hechos fortuitos, por ejemplo, "no me respondió un mensaje, pues seguramente está hablando con otro hombre". El primer punto fue hacerle consiente de que sus ideas incluían a otros posibles hombres como rivales, en un principio comentaba vaguedades a manera de inconformidad, pero no se hacía responsable de dichas ideas: "Seguro estaría hablando con quien sabe quién". Al confrontar sus propias cogniciones y contrastarlas con las evidencias reales, tanto del accionar de su novia como de su madre, los síntomas celopáticos se convirtieron, para Diego, en absurdos, fueron una carga a la que se podía renunciar con el esfuerzo adecuado
Psicoanálisis

Diego se mostró altamente resistente en casi todo el proceso de terapia, resistencia que fue manejada principalmente mediante el uso de la contratransferencia. Diego depositaba en el terapeuta la figura del "padre bueno", una especie de guía que le aconsejara y aceptara incondicionalmente; durante esta fase se hizo patente el tipo de objeto materno que introyectó, objeto ambivalente: a quien ama y al mismo tiempo desprecia. El episodio de infidelidad materna pareció ser un evento lo suficientemente traumático como para determinar la valencia de sus relaciones de pareja

En este contexto, su relación de noviazgo servía como objetivo para desplazar su encono y angustia; la celotipia servía como forma de "castigo" a las mujeres en general, a quienes concebía como dominadas por sus impulsos, personas de gran valía, pero a quienes tenía que vigilarse

Las intervenciones terapéuticas fueron encaminadas a la reparación objetal, enfrentar el episodio de infidelidad materna como un evento externo a él, un conflicto entre adultos que sus padres manejaron como pudieron. Esto con ayuda de la contratransferencia, y la tríada diagnóstica confrontaciónaclaración-interpretación. De esta manera, sus introyectos parentales fueron haciéndose más conscientes conforme avanzaba el proceso y evolucionando hacia la integración de sus objetos de una manera total, ajeno a la disociación en la que se encontraba 


\section{Tabla 1}

\section{Continuación}

Reestructuración cognitiva

En esta fase, los síntomas celotípicos descendieron de manera gradual, pero sistemática, las distorsiones cognitivas, como la sobre generalización y visión de túnel que perpetuaban los celos patológicos, fueron cediendo a la exposición de la evidencia y el pensamiento racional, de tal manera que sus cogniciones fueron ganando, fueron siendo más adaptativas y, en consecuencia, sus emociones y acciones compartieron esa sintonía. Aunado a esto, sucedió que la novia de Diego (Elena) decidió terminar la relación de noviazgo; esto fue relevante por dos cuestiones, en primer término, se hizo patente que Elena de alguna manera estaba siendo gratificada por la neurosis de Diego, gratificación que cesó al remitir los síntomas celotípicos, por otra parte, Diego toleró de muy buena forma la ruptura, al vivir un duelo típico, sin desestructurarse cognitiva ni emocionalmente

Posterior a esta ruptura, Diego se mostró particularmente ávido por establecer alguna relación de pareja nueva; empezó a salir con otras mujeres, pero sin concretar nada formal, al mismo tiempo se mostraba seriamente preocupado por las amistades de su hermana y por la conducta de su madre (temía que de nuevo fuera infiel), sin embargo, las consecuencias de estas preocupaciones fueron menos intensas, según lo reportado en la entrevista, que los síntomas iniciales y era evidente que se esforzaba por establecer patrones de pensamiento saludables y estas preocupaciones fueron cediendo paulatinamente

\section{Cierre}

Diego abandonó el proceso de terapia de manera gradual, en un principio cancelaba una sesión y asistía a la siguiente, de alguna manera intentando espaciar su tratamiento, pero sin reconocerlo abiertamente aun a pesar de la confrontación. Diego a menudo mencionaba que al ya no estar en la relación de noviazgo anterior y tener pocos episodios de celos, se sentía lo suficientemente fuerte como para ir dejando la terapia, aunque abortó el proceso antes de poder tener un cierre formal

\section{Psicoanálisis}

En esta fase, los síntomas celotípicos más superficiales, ya habían remitido, coincidiendo con el fin de su relación de noviazgo. Al confrontar sus conflictos parentales, Diego dejó de desplazar su resentimiento hacia su madre, lo cual hizo que se rompiera la vinculación neurótica con Elena, quien reaccionó de manera violenta ante esta desvinculación y decidió terminar la relación. Diego se mostró particularmente tranquilo, muestra de una fortaleza yoica más desarrollada, pudo atender asuntos académicos y retomar amistades

Sin embargo, su energía se vio redirecccionada, al celar ahora a su hermana y de nuevo a su madre, re-editando el conflicto adolescente, pero ahora reclamando a su madre y vigilándola, recordando el repudio que le causóque en su momento su padre no se hubiera puesto firme y cortado de tajo la aventura de su esposa. No obstante, Diego fue capaz de entender, tanto que estos celos tenían un carácter ilusorio como el proceso mediante el cual se repetía su conflicto. La celotipia está teóricamente vinculada a impulsos de carácter homosexual, no reconocidos por el sujeto, los cuales son proyectados hacia su pareja para aliviar la angustia que le representan. Diego comentó acerca de los hombres con quienes solía celar a Elena, mencionando características que, a manera de insight, asumió que pudieran ser cualidades que a él le pudieran ser atractivas, las intervenciones se enfocaron en reducir su angustia, sin embargo, la sesión posterior fue la última a la que asistió 


\section{Resultados}

Los principales logros de este proceso se establecieron en función de las conductas celotípicas de Diego, quien fue capaz de tolerar la frustración, externalizar sus emociones, detectar las creencias irracionales -las cuales se caracterizan por generar consecuencias de tipo afectivo o conductual que son contraproducentes para sus objetivos y su bienestar-, así como vincularse objetalmente con el terapeuta, establecer asociaciones pertinentes a su caso, crear constancia, vínculos de confianza básica e iniciar la consecución de la totalización del objeto. Para su mejor comprensión, se dividirán los resultados terapéuticos por áreas:

Personal. Esta área, al principio del proceso estaba enmarcada en un problema de confianza básica. Diego mantenía relaciones de suma desconfianza, tanto para sus objetos principales como para el enfrentamiento de retos laborales, académicos y sociales; solía atribuir sus logros a la suerte y sus fracasos a sí mismo. Se encontraba de manera negativa fijado en una imagen de sus objetos parentales inestables, emocionalmente lejanos y poco comprometidos con su familia. A nivel personal esto le generaba una baja capacidad de logro y locus de control externo. A lo largo de las sesiones reportadas, Diego alcanzó un grado importante de reconocimiento de sus propias capacidades, pudo identificar su papel en la consecución de objetivos, aun cuando éstos parecieran casuales, consideró la importancia de recibir afecto, atenciones y estímulos de personas importantes en su vida, situación que en un principio le generaba culpa $\mathrm{y}$ en ocasiones angustia.

A nivel cognitivo, logró establecer la importancia de mantener pensamientos racionales para tener emociones y conductas adaptativas y saludables, aprendió a identificar pensamientos demandantes y pensamientos automáticos para después confrontarlos $\mathrm{y}$ establecer cogniciones alternativas. A nivel dinámico, pudo vincularse transferencialmente con la imagen del terapeuta, lo cual le permitió rescatar su relación objetal paterna; consiguió la capacidad de logro necesaria para concluir su licenciatura, establecer relaciones sociales satisfactorias y significativas y planear su futuro laboral de manera realista y con un grado de ambición importante.

Familiar. Esta área fue la que más afectación mostró. A lo largo del proceso terapéutico, Diego mostró una tendencia evitativa, signo del tipo de vínculo establecido. Sin embargo, en las sesiones finales sus resistencias y rechazo hacia su propio núcleo familiar se vieron debilitadas y logró tener un acercamiento inicial hacia casi todos los miembros de su familia, inició una tentativa de comunicación con sus padres, principalmente, con su madre, logró relativizar moderadamente el episodio de infidelidad materna que en su momento rompió su equilibrio familiar. Un punto importante en su tratamiento fue poder establecer una asociación entre sus impulsos castigadores y controladores hacia su madre y sus conductas celotípicas. Logró avances significativos en el control de los 
impulsos agresivos y pensamientos suspicaces hacia su madre.

De pareja. Ésta es el área detectada por Diego como el motivo de consulta. Los alcances en este tópico fueron variados, aunque no lineales, es decir, los cambios se manifestaban, empero, por cada dos pasos hacia adelante se presentaba uno hacia atrás. Al final de las sesiones reportadas, Diego experimentó una mejoría importante en sus relaciones de pareja. En primera instancia, pudo comprender la imposibilidad de cambiar la discreción a otra persona, sólo por el hecho de desearlo. Aprendió de forma mesurada a tolerar la ambigüedad y el derecho ajeno, a tomar decisiones propias. Logró abrir sus intereses hacia personas distintas a Elena, y analizar la relación de simbiosis hostil que generaba una gran cantidad de angustia $y$ estrés, manteniendo una dinámica subyacente que estimulaban y mantenían las conductas y pensamientos celotípicos. De igual manera, estableció la posibilidad de estar sin pareja y no desestructurarse. Redujo drásticamente sus conductas celotípicas y en un elemento de suma importancia, logró establecer un vínculo entre las conductas castigadoras que ejercía hacia su novia y los impulsos agresivos latentes hacia su madre. Al término de las sesiones, Diego y Elena mantenían una relación cercana sin establecerse de nuevo como pareja. Las conductas de celos por parte de Diego fueron escasas, logró analizarlas y, en muchas ocasiones, estableció cogniciones alternativas.

\section{Discusión y conclusiones}

Diego respondió de manera favorable a la combinación de estrategias terapéuticas sin que éstas se excluyeran mutuamente. Con respecto a la combinación de los enfoques terapéuticos, se pudo llegar a las siguientes conclusiones:

El uso de estrategias terapéuticas combinadas, al abordar casi al mismo tiempo un fenómeno desde perspectivas diferentes, optimiza el proceso, tanto de análisis y consiguiente traslaboración como de reestructuración cognitiva, permitiendo de esta manera una recuperación integral y la remisión de síntomas neuróticos.

Las estrategias terapéuticas cognitivas tienen un efecto fortalecedor del yo, que redunda en una mejoría considerable y una mayor posibilidad de llegar a la sublimación y la traslaboración.

El proceso terapéutico psicodinámico provee de las herramientas necesarias para una adecuada comprensión de las bases históricas de los síntomas disfuncionales, al colaborar con significacia en el proceso de reestructuración cognitiva primordial en la terapia cognitivaconductual.

El uso de estrategias terapéuticas integradas, brinda al clínico una mayor flexibilidad, tanto práctica como teórica y de criterio, siempre en beneficio del paciente y su recuperación. Por otra parte, la combinación de estos dos enfoques conlleva algunos elementos a considerar para una adecuada integración:

- Los enfoques cognitivo y psicodinámico tienen marcadas divergencias en sus conceptos 
y premisas básicas, por lo cual la integración deberá enmarcarse en los puntos convergentes, como son el manejo de las funciones cognitivas como forma de fortalecimiento del yo; la importancia de analizar las experiencias del pasado para trascender las consecuencias desagradables de éstas; la priorización del bienestar y la recuperación del paciente.

- El proceso de transferencia en el proceso terapéutico debe ser cuidado de manera primordial, a fin de que las intervenciones cognitivas no interfieran con la ley de la abstinencia o la atención flotante.

- Es indispensable que el clínico tenga afinidad, conocimiento, facilidad y dominio técnico y teórico amplio de ambos enfoques.

Con respecto al proceso terapéutico, según lo registrado en las entrevistas de cada sesión, se notó una mejoría significativa a lo largo de todo el proceso; los síntomas se modificaron, aproximadamente al inicio de la etapa de tratamiento, aunque, como se citó anteriormente, la remisión no se dio de manera lineal. Las estrategias cognitivas tuvieron un peso importante en la contención emocional ante las circunstancias más estresantes del proceso, en principio frente a la asimilación de los insights logrados. Por su parte, la estructura psicodinámica de las sesiones permitió la reparación del objeto padre, elemento indispensable para este caso en particular; de igual manera, facilitó en el sujeto el desarrollo de habilidades y fortalezas como la confianza, tolerancia a la frustración, empatía, y aceptación.
Finalmente, en cuanto a las limitantes detectadas en el proceso, en primera instancia, se considera la dificultad del sujeto para vincularse afectivamente con sus objetos parentales y como consecuencia con otros objetos en quienes se desplacen estos introyectos. De igual manera, Diego poseía una capacidad de insight un tanto limitada, capacidad que con el paso de las sesiones mejoró de manera sustancial, una marcada compulsión a la repetición, y el desgaste anímico y emocional producto de las disfunciones de su noviazgo, disfunciones que facilitaban la aparición de celos e ideas de tipo paranoicas. La rigidez en su estructura de personalidad impidió que pudiera elaborar por completo los impulsos homosexuales subyacentes a la celotipia, repercutiendo en el abandono del proceso terapéutico y privando de la posibilidad de aplicar por segunda vez las pruebas psicométricas con fin de comparación.

\section{Referencias}

American Psychiatric Association. (2013).

Diagnostic and Statistical Manual of Mental Disorders (DSM-V), (Fifth Edition). Washington, DC: American Psychiatric Association.

Buss, D. M., \& Schmitt, D. (1993). Sexual strategies theory: An evolutionary perspective on human mating. Psychological Review, 100, 204-232.

Carlen, A. M., Kasanzew, A., \& López, A. F. (2009). Tratamiento cognitivo conductual de 
los celos en la pareja. Revista Electrónica de Psicología Iztacala, 12(3), 173-186.

Caudillo, C. \& Cerna, M. (2007) Sexualidad y Vida Humana. México: Universidad Iberoamericana.

Coderch, J. (1990). Teoría y técnica de la psicoterapia psicoanalítica. Barcelona: HERDER.

Cuesta-Bayón, M. T. (2006). Intervención cognitiva en un caso de celotipia. Acción Psicológica, 4(1), 71-82. Recuperadodehttp:// www.redalyc.org/pdf/3440/344030757007. pdf

Ellis, A., \& Blau, S. (2000). Vivir en una sociedad irracional: una guía para el bienestar mediante la terapia racional emotivo-conductual. Barcelona: Paidós.

Esquivel-Sosa, N. Y. \& Gómez-Castillo, B. (2011). Relaciones objetales en un adolescente menor infractor por delito de violación: estudio de caso. Revista Electrónica de Psicología Iztacala, 14(3) 321-348.

Freud, S. (1955). Some neurotic mechanisms in jealousy, paranoia, and homosexuality (Std. ed., Vol. 18). London: Hogarth. (Original workpublished 1922).

Greenson, R. (2004). Técnica y práctica del psicoanálisis. México: Edit. SIGLO XXI.

González, M. \& Herrera, D. (2002). Prevalencia de actitudes celotípicas en estudiantes de dos campus de la UVM. Disponible en http:// www.tlalpan.uvmnet.edu/oiid/download/ Celotipia_04_CSO_PSIC_PICS_E.pdf
Huerta-Hernández， J. N. \& Alcázar-Olán, R. J. (2014). La inteligencia y su relación con las ideas irracionales en estudiantes universitarios. Enseñanza e Investigación en Psicología, 19. Recuperado de http://www. redalyc.org/articulo.oa?id=29238007003

López, F. J. C. (2002). Jealousy: A case of application of Functional Analytic Psychotherapy. Apuntes de Psicología, 20(3), 347-368.

Kernberg, O. (1995). Relaciones amorosas. Normalidad y patología. México: Paidós.

Nasio, J. (1996). Enseñanza de 7 conceptos básicos de Psicoanálisis. Barcelona: Gedisa. Martínez-León, N. C., García-Roncón, L., Barreto-Cortés, D., Alfonso, A., Parra, A., Duque, B., \& Rojas, N. (2013). Características de los celos en un grupo de estudiantes universitarios de la ciudad de Bogotá. Cuadernos Hispanoamericanos de Psicología, 13(1), 36-44.

Palma, B. \& Cosmelli, P. (2008). Aportes de la Psicología y las Neurociencias al concepto del "Insight": la necesidad de un marco integrativo de estudio y desarrollo. Revista Chilena de Neuropsicología, 3, 14-27.

Querol, S., \& Chaves, M. (2009). Test de la persona bajo la lluvia. Buenos Aires: Ed. Lugar.

Rosado-Rosado, M., Vázquez-Vargas, E., \& Cetina-Sosa, A.C. (2016). El Enfoque Integrativo en Psicoterapia. En S. M. AlvaresCuevas, E. M. Escoffié Aguilar, M. Rosado- 
Rosado \& M. Sosa-Correa (Ed.), Terapia en contexto. Una aproximación al Ejercicio Psicoterapéutico. México: Manual Moderno. Sosa-Correa, M., Navarrete-Centeno, J., \& Escoffié-Aguilar, E. M. (2014). El modelo de habilidad de la inteligencia emocional y creatividad. En U. C. de M. Umbral, Conacyt (Ed.), Investigación educativa; estrategias y medios en las universidades de México (pp. 103-114). Recuperado de https:/www. researchgate.net/publication/313890309 EL_MODELO_DE_HABILIDAD_DE_ LA_INTELIGENCIA_EMOCIONAL_Y CREATIVIDAD.

Xandró, M., \& Belda, T. (2007). Test de Machover, pareja y familia. Madrid: Edit. EOS.

Young, J. (1999). Cognitive therapy for personality disorders: a schema - focused approach. Sarasota, FL: Professional Resource Press.

Young, J., \& Klosko, J. (2003). Reinventa tu vida. México: Paidós.

Recibido el 11 de mayo de 2017

Revisado el 06 de julio de 2017

Aceptado el 05 de agosto de 2017 